Korean J. Math. 21 (2013), No. 4, pp. 429-437

http://dx.doi.org/10.11568/kjm.2013.21.4.429

\title{
SEQUENTIAL INTERVAL ESTIMATION FOR THE EXPONENTIAL HAZARD RATE WHEN THE LOSS FUNCTION IS STRICTLY CONVEX
}

\author{
YU SEON JANG
}

\begin{abstract}
Let $X_{1}, X_{2}, \cdots, X_{n}$ be independent and identically distributed random variables having common exponential density with unknown mean $\mu$. In the sequential confidence interval estimation for the exponential hazard rate $\theta=1 / \mu$, when the loss function is strictly convex, the following stopping rule is proposed with the half length $d$ of prescribed confidence interval $I_{n}$ for the parameter $\theta$;

$$
\tau=\text { smallest integer } n \text { such that } n \geq z_{\alpha / 2}^{2} \widehat{\theta}^{2} / d^{2}+2,
$$

where $\hat{\theta}=(n-1) \bar{X}_{n}{ }^{-1} / n$ is the minimum risk estimator for $\theta$ and $z_{\alpha / 2}$ is defined by $P(|Z| \leq \alpha / 2)=1-\alpha(\alpha \in(0,1))$ with $Z \sim N(0,1)$. For the confidence intervals $I_{n}$ which is required to satisfy $P\left(\theta \in I_{n}\right) \geq 1-\alpha$. These estimated intervals $I_{\tau}$ have the asymptotic consistency of the sequential procedure;$$
\lim _{d \rightarrow 0} P\left(\theta \in I_{\tau}\right)=1-\alpha,
$$

where $\alpha \in(0,1)$ is given.

Received September 2, 2013. Revised November 28, 2013. Accepted November $28,2013$.

2010 Mathematics Subject Classification: Primary 62H10, 62H12, $62 \mathrm{H} 15$.

Key words and phrases: sequential estimation, exponential hazard rate, coverage probability.

(c) The Kangwon-Kyungki Mathematical Society, 2013.

This is an Open Access article distributed under the terms of the Creative commons Attribution Non-Commercial License (http://creativecommons.org/licenses/by -nc/3.0/) which permits unrestricted non-commercial use, distribution and reproduction in any medium, provided the original work is properly cited. 


\section{Introduction}

The exponential distribution is often used to model the time between independent events that happen at a constant average rate. In application, the exponential distribution can be used to model lifetimes of various practical situations including but not limited to lengths of times between successive catastrophic events and lengths of time between emergency arrivals at a hospital, to cite a few $[7,10]$.

Let $X_{1}, X_{2}, \cdots, X_{n}$ be independent and identically distributed (IID) random variables having common exponential probability density function (PDF) with unknown mean $\mu$ which is given by

$$
f_{\mu}(x)=\frac{1}{\mu} e^{-x / \mu} \times \mathrm{I}_{(0, \infty)}(x),
$$

where $\mathrm{I}_{A}(\cdot)$ is the indicator function on the set $A$. The hazard function $h(t)$ of a random variable $X$ at time $t$ is defined by

$$
h(t)=\lim _{\Delta t \rightarrow 0} \frac{P\{t \leq X<t+\Delta t \mid X \geq t\}}{\Delta t} .
$$

In the exponential case, the hazard function is represented by the inverse of the mean;

$$
h(t)=\frac{f_{\mu}(t)}{\int_{t}^{\infty} f_{\mu}(x) d x}=\frac{1}{\mu} \equiv \theta, \text { say. }
$$

Models with constant hazard functions are unique and are often useful as baseline distributions to which other distributions are compared or as simple models for failure modes resulting in random failures. The exponential distribution can be characterized as the only distribution with a constant hazard rate $[7,10]$.

Estimation for the parameter is one of the most common forms of statistical inference. One measures a physical quantity in order to estimate its value [6]. The estimator $\widehat{\theta}$ is to be close to $\theta$, we shall interpret this to mean that it will be close on the average. To make this requirement precise, it is necessary to specify a measure of the average closeness of an estimate to $\theta$. The accuracy of an estimator $\widehat{\theta}$ is measured by the risk function

$$
R(\theta, \widehat{\theta})=E_{\theta}\{L(\theta, \widehat{\theta})\}
$$


where $L$ is some loss function, for example, $L(\theta, \widehat{\theta})=(\theta-\widehat{\theta})^{2}+c n$ for the cost $c$ per unit. The best estimator is $\widehat{\theta}$ which minimizes the risk for all $\theta$. But there exists no uniformly best estimator. Sometimes the maximimu likelihood estimator (MLE) or the uniformly minimum variance unbiased estimator (UMVUE) is used as the good estimator of $\theta$. When the loss function is strictly convex, the UMVUE for the parameter $\theta$ is the minimum risk estimator (MRE) by the Rao-Blackwell Theorem.

Takada [9] pointed out that fixed sample size procedure are not available for scale families. Thus, it is necessary to find a sequential sampling rule. Juhlin [4] studied the sequential estimation for the exponential mean parameter and Junvie [7] proposed the sequential confidence interval estimation for the exponential hazard rate using the MLE.

In this paper, estimating sequential confidence intervals for the exponential hazard rate $\theta=1 / \mu$, when the loss function is strictly convex, the following stopping rule is proposed with the half length $d$ of prescribed confidence interval $I_{n}$ for the parameter $\theta$;

$$
\tau=\text { smallest integer } n \text { such that } n \geq z_{\alpha / 2}^{2} \widehat{\theta}^{2} / d^{2}+2,
$$

where $\widehat{\theta}$ is the minimum risk estimator for the $\theta$ and $z_{\alpha / 2}$ is defined by $P(|Z| \leq \alpha / 2)=1-\alpha(\alpha \in(0,1))$ with $Z \sim N(0,1)$. For the confidence intervals $I_{n}$ which is required to satisfy $P\left(\theta \in I_{n}\right) \geq 1-\alpha$, These estimated intervals $I_{\tau}$ have the asymptotic consistency of the sequential procedure;

$$
\lim _{d \rightarrow 0} P\left(\theta \in I_{\tau}\right)=1-\alpha,
$$

where $\alpha \in(0,1)$ is given.

\section{Main Results}

A sequence of random variables $\left\{X_{n}\right\}$ has asymptotically normal with mean $\mu_{n}$ and variance $\sigma_{n}^{2}$, briefly $X_{n} \sim A N\left(\mu_{n}, \sigma_{n}^{2}\right)$, if $\sigma_{n}^{2}>0$ for all $n$ sufficiently large and

$$
\frac{X_{n}-\mu_{n}}{\sigma_{n}} \stackrel{\mathscr{D}}{\rightarrow} Z \sim N(0,1)
$$

where $\stackrel{\mathscr{D}}{\rightarrow}$ stands for the convergence in distribution. The following lemma comes from Serfling [8]. 
Lemma 2.1. If $X_{n} \sim A N\left(\mu_{n}, \sigma_{n}^{2}\right)$, then also $a_{n} X_{n}+b_{n} \sim A N\left(\mu_{n}, \sigma_{n}^{2}\right)$ if and only if

$$
a_{n} \rightarrow 1, \frac{\mu_{n}\left(a_{n}-1\right)+b_{n}}{\sigma_{n}} \rightarrow 0 \text { as } n \rightarrow \infty .
$$

In particular, if $\mu_{n}=0, \sigma_{n}^{2}=1$, then $\left|a_{n}\right|$ instead $a_{n} \rightarrow 1$.

Theorem 2.2. Let $X_{1}, X_{2}, \cdots, X_{n}$ be IID exponential random variables with hazard rate $\theta$ and let $T_{n}=(n-1) \bar{X}_{n}^{-1} / n$. Then $T_{n}$ is the UMVUE for $\theta$ and $T_{n}$ has $A N\left(\theta, \theta^{2} /(n-2)\right)$.

Proof. First, we prove that $T_{n}$ is the UMVUE for $\theta$. Since the complete sufficient statistic $\bar{X}_{n}$ is unbiased, $\bar{X}_{n}$ is the UMVUE. From Lehmann-Sheffe's Theorem it suffices to show that $T_{n}$ is an unbiased estimator of $\theta$. Since $S=\sum_{i=1}^{n} X_{i}$ has the Erlang distribution, $\operatorname{Erlang}(n, \theta)$, thus we have

$$
E_{\theta}\left(T_{n}\right)=(n-1) \int_{0}^{\infty} \frac{1}{s \Gamma(n)} \theta^{n} s^{n-1} e^{-\theta s} d s=\theta
$$

because $\theta^{n-1} s^{n-2} e^{-\theta s} / \Gamma(n-1)$ is the PDF of Erlang distribution, Erlang $(n-1, \theta)$. Next, we prove the asymptotic normality of $T_{n}$. Now, the variance of $T_{n}$ is

$$
\operatorname{Var}\left(T_{n}\right)=(n-1)^{2} \int_{0}^{\infty} \frac{1}{s^{2} \Gamma(n)} \theta^{n} s^{n-1} e^{-\theta s} d s-\theta^{2}=\frac{\theta^{2}}{n-2} .
$$

Since $\bar{X}_{n}^{-1}$ is MLE of $\theta$, it follows asymptotic normality. From the Lemma 2.1 we obtain that

$$
T_{n}=\frac{(n-1)}{n} \bar{X}_{n}^{-1} \sim A N\left(\theta, \frac{\theta^{2}}{n-2}\right) .
$$

This proof is complete.

From Rao-Blackwell's Theorem we have the following corollary.

Corollary 2.3. When the loss function is strictly convex, the UMVUE $T_{n}$ for $\theta$ in Theorem 2.2 is the MRE.

For a good estimator $\widehat{\theta}$, let $I_{n}=[\widehat{\theta}-d, \widehat{\theta}+d]$ be a confidence interval for $\theta$ with confidence coefficient $1-\alpha$, where $d>0$ and $0<\alpha<1$, that is,

$$
P\left(\theta \in I_{n}\right)=1-\alpha
$$


Now, the coverage probability for $\theta$ with $I_{n}: T_{n} \pm d(d>0)$ as the confidence interval is given by $P\left\{\theta \in I_{n}\right\} \geq 1-\alpha$.

Set

$$
n^{*}=\frac{z_{\alpha / 2}^{2}}{d^{2}} \theta^{2}+2
$$

Since for sufficiently large $n$

$$
P\left\{\frac{\left|T_{n}-\theta\right|}{\theta / \sqrt{n-2}} \leq z_{\alpha / 2}\right\} \approx 1-\alpha,
$$

we then have

$$
P\left\{\theta \in I_{n}\right\} \geq P\left\{\left|T_{n}-\theta\right| \leq \frac{z_{\alpha / 2}}{\sqrt{n-2}} \theta\right\} \approx 1-\alpha .
$$

Since $\theta$ is unknown, $n^{*}$ is also unknown. In sequential estimating the confidence interval $I_{n}$, consider the following stopping rule:

$$
\tau=\text { smallest integer } n \text { such that } n \geq \frac{z_{\alpha / 2}^{2}}{d^{2}} T_{n}^{2}+2,
$$

where $T_{n}=(n-1) \bar{X}^{-1} / n$ is the UMVUE of $\theta$.

From the Chow-Robbins procedure [1], one has the following results.

Lemma 2.4. Let $n^{*}$ and $\tau$ be defined as in (2.7) and (2.10), respectively. Then the following statements hold:

(1) $P\{\tau<\infty\}=1$ for all $d>0$,

(2) $\tau \rightarrow \infty$ with probability 1 as $d \rightarrow 0$,

(3) $\frac{\tau}{n^{*}} \rightarrow 1$ with probability 1 as $d \rightarrow 0$.

Lemma 2.5. Let $\tau$ be defined as in (2.10). Then

$$
\frac{T_{\tau}-\theta}{\theta / \sqrt{\tau-2}} \stackrel{\mathscr{P}}{\rightarrow} N(0,1) \text { as } d \rightarrow 0 .
$$

Proof. For all $\mu>0$, by the Taylor expansion of $h(x)=(n-1) / n x$ at $x=\mu$, we have

$$
T_{n}=\frac{n-1}{n} \theta-\frac{n-1}{n} \theta^{2}\left(\bar{X}_{n}-\mu\right)+R_{n}
$$


where $R_{n}=(n-1)\left(\bar{X}_{n}-\mu\right)^{2} / 2 n \xi^{2}$ and $\xi$ is a random variable lying between $\bar{X}_{n}$ and $\mu$. This yields

$$
\frac{T_{n}-\theta}{\theta / \sqrt{n-2}}=a_{n} Z_{n}+b_{n}+R_{n}^{*}
$$

where $a_{n}=-(n-1) \sqrt{n-2} / n \sqrt{n}, b_{n}=-\sqrt{n-2} / n$, $Z_{n}=\sqrt{n}\left(\bar{X}_{n}-\mu\right) / \mu$, and

$$
\left|R^{*}\left(\bar{X}_{n}, \xi\right)\right| \leq \frac{M\left|\bar{X}_{n}-\xi\right|^{2}}{2}
$$

for some $M<\infty$. By the Central Limit Theorem, $Z_{n} \stackrel{\mathscr{D}}{\rightarrow} Z \sim N(0,1)$. From Lemma 1.1 we know that

$$
a_{n} Z_{n}+b_{n} \stackrel{\mathscr{D}}{\rightarrow} N(0,1) \text { as } n \rightarrow \infty .
$$

Now, $\tau \rightarrow \infty$ as $d \rightarrow 0$. Since $a_{n} Z_{n}+b_{n}$ is uniform continuity in probability and stochastically bounded from Woodroofe [12], thus we have

$$
a_{\tau} Z_{\tau}+b_{\tau} \stackrel{\mathscr{D}}{\rightarrow} Z \sim N(0,1) \text { as } d \rightarrow 0,
$$

From the Strong Law of Large Numbers $\left|\bar{X}_{\tau}-\theta\right| \rightarrow 0$ with probability 1. As result, $R_{\tau}^{*} \rightarrow 0$ in probability as $d \rightarrow 0$. Consequently, from the Slutsky's Theorem the proof of this lemma is complete.

THEOREM 2.6. When the loss function is strictly convex, let $\tau$ be defined as in (2.10). Then

$$
\lim _{d \rightarrow 0} P\left\{\theta \in I_{\tau}\right\}=1-\alpha .
$$

Proof. From the definition of $\tau$ in (2.10), we have

$$
d \sqrt{\tau-2} / \theta \geq z_{\alpha / 2}
$$

and

$$
P\left\{\theta \in I_{\tau}\right\}=P\left\{\frac{\left|T_{\tau}-\theta\right|}{\theta / \sqrt{\tau-2}} \leq \frac{d \sqrt{\tau-2}}{\theta}\right\} \geq 1-\alpha
$$

Set

$$
Z_{\tau}=\frac{T_{\tau}-\theta}{\theta / \sqrt{\tau-2}}
$$


For any fixed $\varepsilon>0$,

$$
\begin{aligned}
P\left\{\left|Z_{\tau}\right| \leq \frac{z_{\alpha / 2} T_{\tau}}{\theta}\right\}= & P\left\{\left|Z_{\tau}\right| \leq \frac{z_{\alpha / 2} T_{\tau}}{\theta},\left|\frac{T_{\tau}}{\theta}-1\right| \leq \varepsilon\right\} \\
& +P\left\{\left|Z_{\tau}\right| \leq \frac{z_{\alpha / 2} T_{\tau}}{\theta},\left|\frac{T_{\tau}}{\theta}-1\right|>\varepsilon\right\} \\
\leq P & \left\{\left|Z_{\tau}\right| \leq \frac{z_{\alpha / 2} T_{\tau}}{\theta},\left|\frac{T_{\tau}}{\theta}-1\right| \leq \varepsilon\right\} \\
& +P\left\{\left|\frac{T_{\tau}}{\theta}-1\right|>\varepsilon\right\} \\
\leq & P\left\{\left|Z_{\tau}\right| \leq z_{\alpha / 2}(1+\varepsilon)\right\}+P\left\{\left|\frac{T_{\tau}}{\theta}-1\right|>\varepsilon\right\} .
\end{aligned}
$$

By the Strong Law of Large Numbers, $T_{\tau} \rightarrow \theta$ with probability 1 as $d \rightarrow 0$. Hence, $T_{\tau} / \theta \rightarrow 1$ in probability as $d \rightarrow 0$ and

$$
\lim _{\varepsilon \rightarrow 0} P\left\{\left|\frac{T_{\tau}}{\theta}-1\right|>\varepsilon\right\}=0
$$

Now, by Lemma $1.2, Z_{\tau} \stackrel{\mathscr{D}}{\rightarrow} Z \sim N(0,1)$ as $d \rightarrow 0$. Thus, letting $d \rightarrow 0$ and taking $\varepsilon \rightarrow 0$ we have

$$
\limsup _{d \rightarrow 0} P\left\{\left|Z_{\tau}\right| \leq \frac{z_{\alpha / 2} T_{\tau}}{\theta}\right\} \leq 1-\varepsilon .
$$

Similarly, we have

$$
\begin{aligned}
P\left\{\left|Z_{\tau}\right| \leq \frac{z_{\alpha / 2} T_{\tau}}{\theta}\right\}= & P\left\{\left|Z_{\tau}\right| \leq \frac{z_{\alpha / 2} T_{\tau}}{\theta},\left|\frac{T_{\tau}}{\theta}-1\right| \leq \varepsilon\right\} \\
& -P\left\{\left|\frac{T_{\tau}}{\theta}-1\right|>\varepsilon\right\} \\
& +P\left\{\left|Z_{\tau}\right| \leq \frac{z_{\alpha / 2} T_{\tau}}{\theta},\left|\frac{T_{\tau}}{\theta}-1\right|>\varepsilon\right\} \\
& +P\left\{\left|\frac{T_{\tau}}{\theta}-1\right|>\varepsilon\right\} \\
\geq & P\left\{\left|Z_{\tau}\right| \leq z_{\alpha / 2}(1-\varepsilon)\right\}-P\left\{\left|\frac{T_{\tau}}{\theta}-1\right|>\varepsilon\right\} .
\end{aligned}
$$


Hence, taking $\varepsilon \rightarrow 0$ we get

$$
\liminf _{d \rightarrow 0} P\left\{\left|Z_{\tau}\right| \leq \frac{z_{\alpha / 2} T_{\tau}}{\theta}\right\} \geq 1-\alpha
$$

and thus we have

$$
\lim _{d \rightarrow 0} P\left\{\left|Z_{\tau}\right| \leq \frac{z_{\alpha / 2} T_{\tau}}{\theta}\right\} \geq 1-\alpha
$$

As a result, $\liminf _{d \rightarrow 0} P\left\{\theta \in I_{\tau}\right\} \geq 1-\alpha$. Now, observe that

For any $\varepsilon>0$,

$$
\begin{gathered}
\tau-1<\frac{z_{\alpha / 2}^{2}}{d^{2}} T_{\tau-1}^{2}+2 \\
\frac{d}{\theta z_{\alpha / 2}} \sqrt{\tau}<\sqrt{\frac{T_{n}^{2}}{\theta^{2}}+\frac{d^{2}}{\theta^{2} z_{\alpha / 2}^{2}}} \equiv k_{d}, \quad \text { say }
\end{gathered}
$$

$$
\begin{aligned}
P\left\{\theta \in I_{\tau}\right\} \leq & P\left\{\left|Z_{\tau}\right| \leq z_{\alpha / 2} k_{d},\left|k_{d}-1\right| \leq \varepsilon\right\} \\
& +P\left\{\left|Z_{\tau}\right| \leq z_{\alpha / 2} k_{d},\left|k_{d}-1\right|>\varepsilon\right\} \\
\leq & P\left\{\left|Z_{\tau}\right| \leq z_{\alpha / 2}(1+\varepsilon)\right\}+P\left\{\left|k_{d}-1\right|>\varepsilon\right\} .
\end{aligned}
$$

By the Strong Law of Large Number $k_{d} \rightarrow 1$ with probability 1 as $d \rightarrow 0$.

Taking $\varepsilon \rightarrow 0$, it follows that

$$
\limsup _{d \rightarrow 0} P\left\{\theta \in I_{\tau}\right\} \leq 1-\alpha .
$$

Therefore the proof is complete.

\section{References}

[1] Y. S. Chow and H. Robbins, On the asymptotic theory of fixed width sequential confidence intervals for the mean, Ann. Math. Stat. 36 (1965), 457-462.

[2] M. Ghosh, N. Mukhopadhyay, and P. K. Sen, Sequential Estimation, Wiley, 1997.

[3] Y. Takada, Non-existence of fixed sample size procedures for sacle families, Sequential Anal. 5 (1986), 99-100.

[4] K. D. Juhlin, Sequential and non-sequential confidence intervals with guaranteed coverage probability and beta-protection, $\mathrm{PhD}$ Dissertation, University of Illinois, 1985.

[5] S. M. Kay, Fundamentals of Statistical Signal Processing, Prentice Hall PTR, 1993.

[6] E. L. Lehmann and G. Cassella, The Theory of Point Estimation, 2nd edition, Springer, 1998. 
[7] J. M. Pailden and D. L. L. Polestico, Sequential Confidence Intervals for the Exponential Hazard Rate, 10th National Convention on Statistics (NCS) Oct. $1-2,2007$.

[8] R. J. Serfling, Approximation Theorems of Mathematical Statistics, Wiley, 1980.

[9] Y. Takada, Non-existence of fixed sample size procedures for scale families, Sequential Anal. 5 (1986), 99-100.

[10] C. Uno, E. Isogai, and D. L. Lim, Sequential point estimation of a function of the exponential scale parameter, Aust. J. Stat. 33 (2004), 281-291.

[11] R. A. Wijsman, Sequential Confidence Intervals with Beta-Protection in OneParameter Families, Lecture Notes-Monograph Series 8 (1985), 96-107.

[12] M. Woodroofe, Nonlinear renewal theory in sequential analysis, CBMS-NSF Regional Conference Series in Applied Mathematics 39, 1982., SIAM.

Department of Applied Mathematics

Kangnam University

Yongin 446-702, Republic of Korea

E-mail: ysjang@kangnam.ac.kr 